\title{
ROS Scavenger, Ebselen, Has No Preventive Effect in New Hearing Loss Model Using a Cholesterol-Chelating Agent
}

\author{
Min Young Lee ${ }^{1}$, Lisa L. Kabara², Donald L. Swiderski², \\ Yehoash Raphael ${ }^{2}$, R. Keith Duncan², and Young Ho Kim \\ 1 Department of Otorhinolaryngology and Head \& Neck Surgery, Dankook University Hospital, Cheonan, Korea \\ ${ }^{2}$ Kresge Hearing Research Institute, Otolaryngology-Head and Neck Surgery, The University of Michigan, Ann Arbor, MI, USA \\ ${ }^{3}$ Department of Otorhinolaryngology, Boramae Medical Center, Seoul Metropolitan Government-Seoul National University, Seoul, Korea
}

\author{
Received July 12,2018 \\ Revised September 4, 2018 \\ Accepted September 19, 2018
}

\begin{abstract}
Background and Objectives: The antioxidant ebselen will be able to limit or prevent the ototoxicity arising from 2-hydroxypropyl- $\beta$-cyclodextrin (HPBCD). Niemann-Pick Type C (NPC) disease is a disorder of lysosomal storage manifested in sphingolipidosis. Recently, it was noted that experimental use of HPBCD could partially resolve the symptoms in both animals and human patients. Despite its desirable effect, HPßCD can induce hearing loss, which is the only major side effect noted to date. Understanding of the pathophysiology of hearing impairment after administration of HPßCD and further development of preventive methods are essential to reduce the ototoxic side effect. The mechanisms of HPBCD-induced ototoxicity remain unknown, but the resulting pathology bears some resemblance to other ototoxic agents, which involves oxidative stress pathways. To indirectly determine the involvement of oxidative stress in HPßCD-induced ototoxicity, we tested the efficacy of an antioxidant reagent, ebselen, on the extent of inner ear side effects caused by HPRCD. Materials and Methods: Ebselen was applied prior to administration of HP $\beta C D$ in mice. Auditory brainstem response thresholds and otopathology were assessed one week later. Bilateral effects of the drug treatments also were examined. Results: HPBCD-alone resulted in bilateral, severe, and selective loss of outer hair cells from base to apex with an abrupt transition between lesions and intact areas. Ebselen co-treatment did not ameliorate HPBCD-induced hearing loss or alter the resulting histopathology. Conclusions: The results indirectly suggest that cochlear damage by HPBCD is unrelated to reactive oxygen species formation. However, further research into the mechanism(s) of HPßCD otopathology is necessary.
\end{abstract}

J Audiol Otol 2019;23(2):69-75

\section{Introduction}

Niemann-Pick Type C (NPC) disease is a lysosomal storage disorder associated with the accumulation of glycosphingolipids and cholesterol in lysosomes. This accumulation of fatty substance or lipid in the multiple organs of the body including liver, spleen, brain and bone, especially in brain, may cause various symptoms such as ataxia, dysarthria, dysphagia, abnormal posturing and in most cases, this leads to a fa-

This is an Open Access article distributed under the terms of the Creative Commons Attribution Non-Commercial License (https://creativecommons.org/licenses/by-nc/4.0/) which permits unrestricted non-commercial use, distribution, and reproduction in any medium, provided the original work is properly cited. tal prognosis. Recently, the experimental administration of 2-hydroxypropyl- $\beta$-cyclodextrin (HP $\beta C D$ ) was shown to be able to resolve some symptoms in both animal and human NPC $[1,2]$. HP $\beta C D$ mobilizes cholesterol as well as phospholipids and proteins, which aids transport of accumulated cholesterol out of the lysosome. When HP $\beta C D$ was used in high doses, it was reported to have ototoxic effects on cochlear outer hair cells (OHCs) in mice [3-6]. However, the biological pathway for this damage remains to be elucidated.

One possible explanation for the loss of OHCs and resultant hearing impairment made by $\mathrm{HP} \beta C D$ is catastrophic damage to the cell membrane by mobilizing or depleting membrane lipids [3]. However, an effect on ubiquitous lipid components 
cannot easily explain the specificity of injury to the OHCs. OHCs show heightened sensitivity to other ototoxic agents, such as cisplatin and aminoglycosides, compared to other cochlear cell types, possibly due to differences in uptake mechanisms [7] and mitochondrial metabolism [8]. Indeed, increased reactive oxygen species (ROS) production is a common cause in otopathology from several forms of acquired hearing loss [9]. Therefore, we sought to examine whether antioxidant therapy could limit or prevent HP $\beta C D$-ototoxicity.

Ebselen, an organoselenium compound, has antioxidant and anti-inflammatory effects [10]. This drug has preventive effects on cisplatin ototoxicity in auditory cells, organotypic cultures, and mouse in vivo. Its effect is thought to be caused by suppressing the production of ROS and intracellular oxidative agents, and increasing the expression of antioxidant responsive element through the $N r f 2$ translocation to the nucleus [11]. The aim of this study was to investigate whether ebselen could ameliorate HP $\beta C D$-induced hearing loss in mice.

\section{Materials and Methods}

\section{Animals and experimental procedures}

Four- to six-week old FVB/NJ mice (20-24 g, n=14) were used in this study. These animals were divided into 3 groups, which are control $(n=2), \operatorname{HP} \beta C D$ alone $(n=6)$, and ebselen plus $\operatorname{HP} \beta C D(n=6)$. Control animals were treated with normal saline (i.p., single). The ebselen dose was $16 \mathrm{mg} / \mathrm{kg}$ (i.p., single), and the HP $\beta C D$ dose was $8,000 \mathrm{mg} / \mathrm{kg}$ (s.c., single) $[3,11]$. In ebselen and HP $\beta C D$ groups, ebselen was administered 1 day before HP $\beta C D$ treatment.

This study was approved (A3114-01) and performed according to the guidelines of the University of Michigan Institutional Animal Care and Use Committee (IACUC).

\section{ABRs recording}

Auditory brainstem responses (ABRs) were recorded before and 1 week after IP injection of ebselen $(12,24 \mathrm{kHz})$. Animals were anesthetized intramuscularly with ketamine $(58.8 \mathrm{mg} / \mathrm{kg})$, xylazine $(2.4 \mathrm{mg} / \mathrm{kg})$, and acepromazine $(1.2$ $\mathrm{mg} / \mathrm{kg}$ ) and placed on a thermo-regulating heating pad to maintain body temperature. ABRs were recorded in an electrically and acoustically shielded chamber (Acoustic Systems, Austin, TX, USA). Tucker Davis Technologies (TDT) System III hardware and SigGen/BioSig software (TDT, Alachua, FL, USA) were used to present the stimulus and record responses. Neural activity in response to brief tone bursts was measured using needle electrodes inserted subcutaneously ventral to each pinna and at the vertex of the skull. The sound was delivered to an area just inside the tragus. Each tone burst was $15 \mathrm{~ms}$ in duration, with $1 \mathrm{~ms}$ rise/fall times, presented 10 bursts per second through an EC1 driver (TDT, aluminum enclosure made in-house). 1,024 responses were averaged for each stimulus level and each frequency. Responses were collected for stimulus levels in $10 \mathrm{~dB}$ steps at higher stimulus levels, and at $5 \mathrm{~dB}$ steps near threshold. Thresholds were interpolated between the lowest stimulus level where a response was observed, and $5 \mathrm{~dB}$ lower, where no response was observed.

\section{Tissue preparation and immunocytochemistry}

After the ABR recordings, epi-fluorescence analysis using primary antibody to prestin (right ear) and ZO-1 (left ear), and Phalloidin was performed. Animals were sacrificed after ABR test and their cochleae were harvested. Samples were fixed in $2 \%$ paraformaldehyde in phosphate buffered saline (PBS) for at least 1 hour, rinsed with PBS, and the area of the auditory epithelium and spiral limbus were dissected for whole-mount preparations. Staining for prestin (right ear) and ZO-1 (left ear) was used to evaluate the integrity of OHC membranes and tight junctional complexes, respectively. The dissected tissues were permeabilized in $0.3 \%$ Triton X-100 in PBS for $10 \mathrm{~min}$, then incubated with blocking buffer $(5 \%$ normal goat serum in PBS) for 30 min to block non-specific binding of secondary antibodies. After that, samples were incubated with primary antibodies. The following primary antibodies, dilutions and incubation conditions were used; antiprestin antibody [sc-22692 (Santa Cruz Biotechnology, Dallas, TX, USA), 1:100 dilution in blocking buffer, $1 \mathrm{~h}$, room conditions]; monoclonal anti-ZO-1 antibody (Zymed Laboratories, Inc., San Francisco, CA, USA, 1:100 dilution in blocking buffer, overnight, at $4^{\circ} \mathrm{C}$ ). After rinsing the primary antibody with PBS, tissues were incubated with a fluorescence-labeled secondary antibody for $30 \mathrm{~min}$ and rinsed with PBS before mounting on microscope slides. To label F-actin, permeabilized dissected tissues were incubated with Alexa Fluor 488conjugated Phalloidin (Abcam, Cambridge, MA, USA, 1:400 dilution in PBS, 30 min, room temperature). After rinsing with PBS, stained tissues were mounted on glass slides with Fluoro-Gel mounting media (Electron Microscopy Sciences, Hatfield, PA, USA). For epi-fluorescence analysis, we used a Leica DMRB epi-fluorescence microscope (Leica, Eaton, PA, USA) equipped with a SPOT-RT digital camera (Diagnostic Instruments, Sterling Heights, MI, USA) and SPOT-RT software Ver.5.0. For confocal microscopy analysis, we used Olympus FV 500 Confocal microscope (Olympus, Center Valley, PA, USA). Photographs were cropped and labeled with Adobe Photoshop and Illustrator software (Adobe System, San Jose, CA, USA). 


\section{Statistical analysis}

All data were analyzed by GraphPad Prism (GraphPad Software, La Jolla, CA, USA) software.

Hearing thresholds were compared between groups (with and without ebselen treatment) and time points (before and after systemic administration of pharmacologic agents) using unpaired Student's T-test. $p$-value $\leq 0.05$ was considered as statistically significant.

\section{Results}

\section{ABRs results}

To evaluate the hearing outcome after injection of HP $\beta C D$ or ebselen or both, $\mathrm{ABR}$ was performed before and 1 week after administration. Baseline ABR thresholds before the injection of drugs did not show significant differences between groups, hearing thresholds are shown in Table 1. ABR thresholds after drug injection were compared to the ABR thresholds of control animals treated with normal saline. The ebselen treatment group had ABR thresholds similar to controls, suggesting that ebselen itself was not ototoxic. Injection of $\mathrm{HP} \beta C D$ resulted in significant hearing loss showing mean thresholds higher than $60 \mathrm{~dB}$ SPL at both $12 \mathrm{kHz}$ and $24 \mathrm{kHz}$,

Table 1. Baseline hearing thresholds of four different groups

\begin{tabular}{|c|c|c|}
\hline & $12 \mathrm{kHz}(\mathrm{dB} S P L \pm S D)$ & $24 \mathrm{kHz}(\mathrm{dB} S P L \pm S D)$ \\
\hline Control & $17.50 \pm 3.54$ & 15.00 \\
\hline $\mathrm{HP \beta CD}$ & $15.00 \pm 1.79$ & $14.17 \pm 3.97$ \\
\hline Ebselen+HP $\beta C D$ & $16.14 \pm 2.97$ & $15.14 \pm 4.88$ \\
\hline
\end{tabular}

SD: standard deviation, HP $\beta C D$ : hydroxypropyl- $\beta$-cyclodextrin which revealed statistically significant differences compared to thresholds of the control group ( $p=0.005$ and 0.02 at 12 and $24 \mathrm{kHz}$, respectively) (Fig. 1). ABR threshold shifts were about $40 \mathrm{~dB}$ in most $\mathrm{HP} \beta \mathrm{CD}$-injected mice, however, threshold shift was not found in all animals, as reported previously. [3] Combined administration of ebselen and $\mathrm{HP} \beta \mathrm{CD}$ also resulted in significantly elevated thresholds compared to the control group at both frequencies $(p=0.03$ and 0.02 at 12 and $24 \mathrm{kHz}$, respectively) (Fig. 1). There was no difference of threshold between HP $\beta C D$ only and the combination of ebselen/HP $\beta C D$ at both frequencies (Fig. 1), suggesting ebselen exerted no protective effect against cytotoxicity of $\mathrm{HP} \beta C D$.

\section{Prestin expression in OHCs after HP $\beta C D$ and ebselen}

Prestin was previously found to be a direct or indirect target in OHCs exposed to HP $\beta C D$, based on drug-induced mislocalization of prestin and the resistance of OHCs to HP $\beta C D$ in prestin-null mice $[3,4]$. We therefore evaluated whether a change in prestin expression was seen after HP $\beta C D$-only and $\mathrm{HP} \beta \mathrm{CD} /$ ebselen treatments in OHCs that survive the insult. Indeed, using prestin-specific immunocytochemistry, we observed differences in the distribution and intensity of prestin staining between control and $\mathrm{HP} \beta \mathrm{CD}$ only treated groups (Fig. 2). Control organ of Corti showed well organized rings of prestin at the perimeter of OHCs whereas cyclodextrin-treated groups showed disrupted prestin organization. Still, the ebselen+HP $\beta C D$ group appeared to have less disruption of prestin compared to HP $\beta C D$ only treated groups (Fig. 2). The damage was restricted to the OHCs; inner hair cells remained intact.
Fig. 1. Mean ABRs threshold after treatment in each group. Mean ABRs threshold in HP $\beta C D$-only group shows a statistically significant elevation compared to that in control group 1 week after administration of HPBCD ( $p=0.005$ and 0.02 at 12 and $24 \mathrm{kHz}$, respectively). Combination group treated with ebselen and $H P B C D$ also has a statistically significant elevation of mean ABRs threshold similar to HPBCD alone group ( $p=0.03$ and 0.02 at 12 and $24 \mathrm{kHz}$, respectively). ABRs threshold shifts were about $40 \mathrm{~dB}$ in most mice treated with HP $\beta C D$ (asterisks: $p<0.05)$. ABRs: auditory brainstem responses, HP $\beta C D$ : hydroxypropyl$\beta$-cyclodextrin.

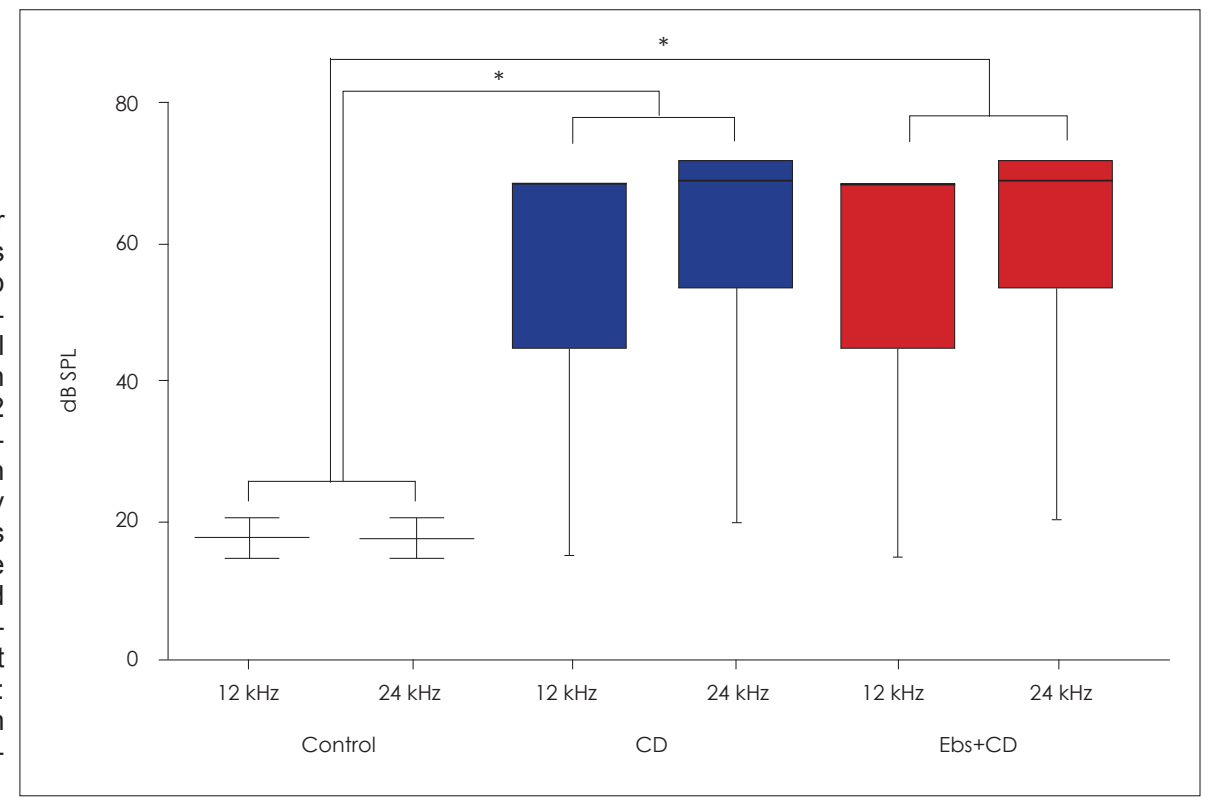


CD alone
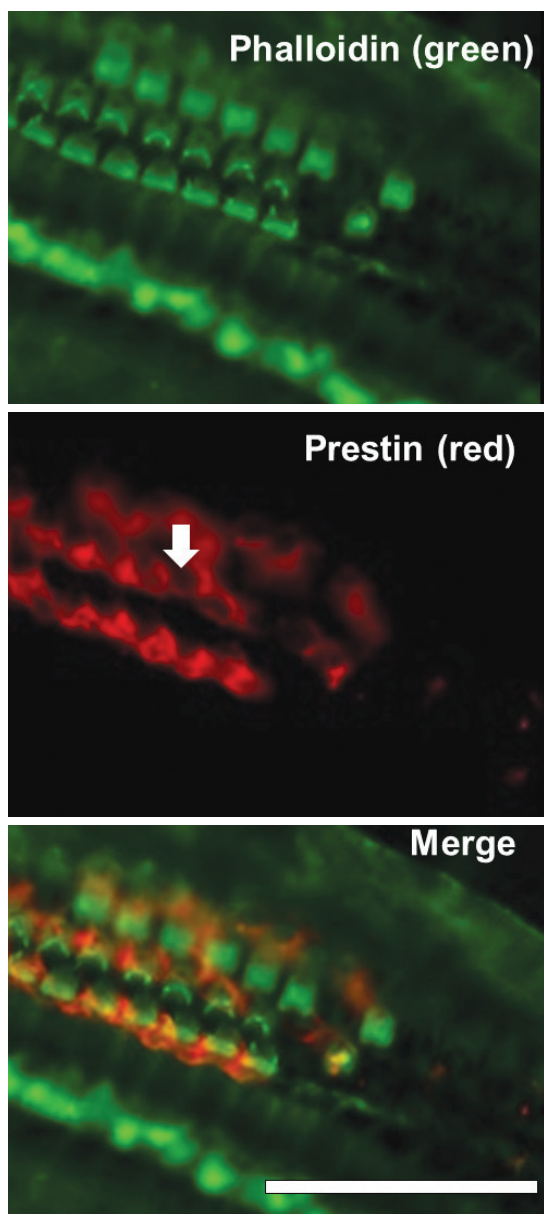

CD (-) "control"
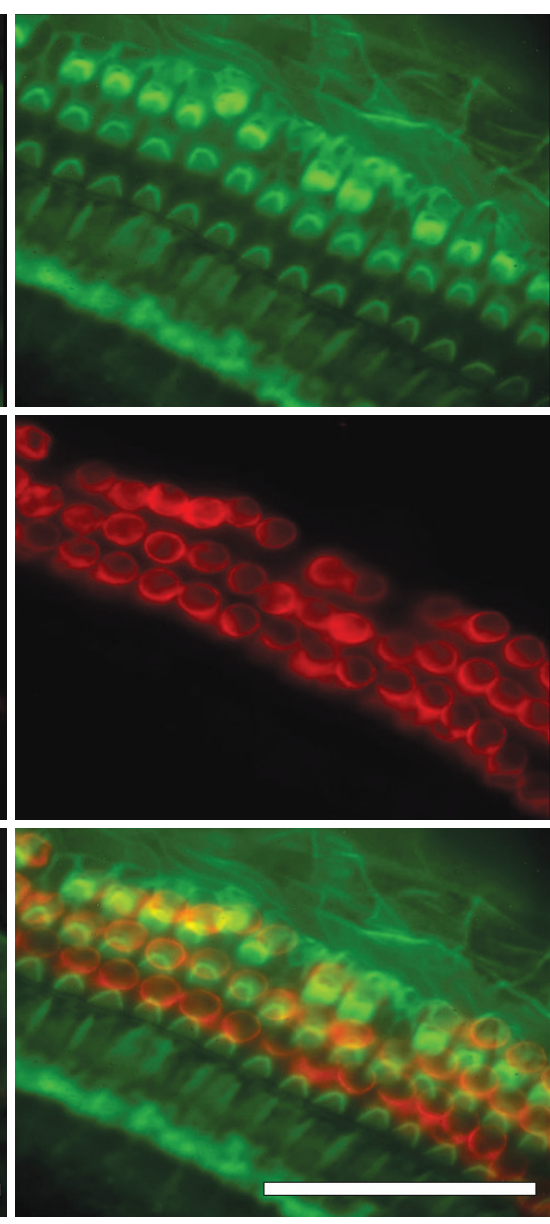

Ebselen $+\mathrm{CD}$
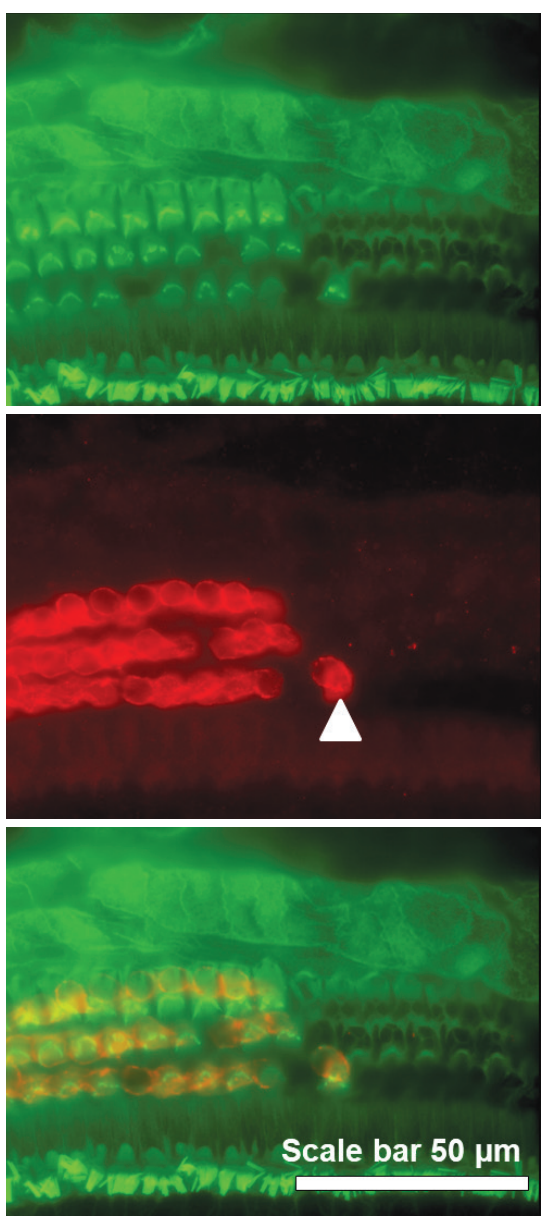

Fig. 2. Epi-fluorescence of whole-mounts of the organ of Corti stained for prestin and phalloidin. Upper row is images stained with Phalloidin and middle low is prestin and bottom row is merged. HPßCD only group (left column) reveals an area with OHC loss and disrupted pattern (arrow) of prestin distribution in remaining OHCs. Control group not treated with HPßCD (middle column) shows normal staining pattern of prestin in the organ of Corti. Combination group treated with ebselen and HP $\beta C D$ (right column) demonstrates some $\mathrm{OHC}$ loss and less disruption of prestin compared to HPBCD only group (arrow head). CD: cyclodextrin, OHCs: cochlear outer hair cells, HPßCD: hydroxypropyl- $\beta$-cyclodextrin.

\section{Histological findings on cochlear epithelial surface}

To evaluate the integrity of tight junctional complexes within the cochlear sensory epithelium one-week after HP $\beta C D$ only or ebselen/HP $\beta C D$ administration, ZO1 staining was used. In the control group without damage by $\mathrm{HP} \beta \mathrm{CD}$, intact tight junctions on the epithelial cells were found. In the cochleae of HP $\beta C D$ only and ebselen/HP $\beta C D$ groups, no disruption of the tight junctional complexes was observed (Fig. 3). These results suggest that $\mathrm{HP} \beta \mathrm{CD}$ does not cause a persistent disruption the tight junctional complexes.

\section{Bilateral symmetry of HPBCD otopathology}

$\mathrm{HP} \beta C D$-only or the combination of ebselen and $\mathrm{HP} \beta C D$ induced $\mathrm{OHC}$ damage. In these two groups, complete loss of OHCs was restricted to the hook and basal turn of the mouse cochlea. In the lower frequency regions (apical cochlea) $\mathrm{OHC}$ survival was nearly complete. The transition zone between the region of severe lesion (base) and full survival of $\mathrm{OHC}$ was very narrow, showing an abrupt transition from almost complete survival to nearly complete loss of OHCs (Fig. 2). This finding is consistent with prior observations [3,5]. The symmetry of the transition zone from death to survival was examined for right and left ears and compared between $\mathrm{HP} \beta C D$ only and ebselen/HP $\beta C D$ treatment groups. HP $\beta C D$-only treated group showed symmetry (all $<45^{\circ}$ ). However, in one out of 5 animals (20\%) in the combination group, the asymmetry was more than a $45^{\circ}$ difference between right and left cochleae (Fig. 4).

\section{Discussion}

In the present study, we demonstrated the pattern of $\mathrm{OHC}$ 
CD alone
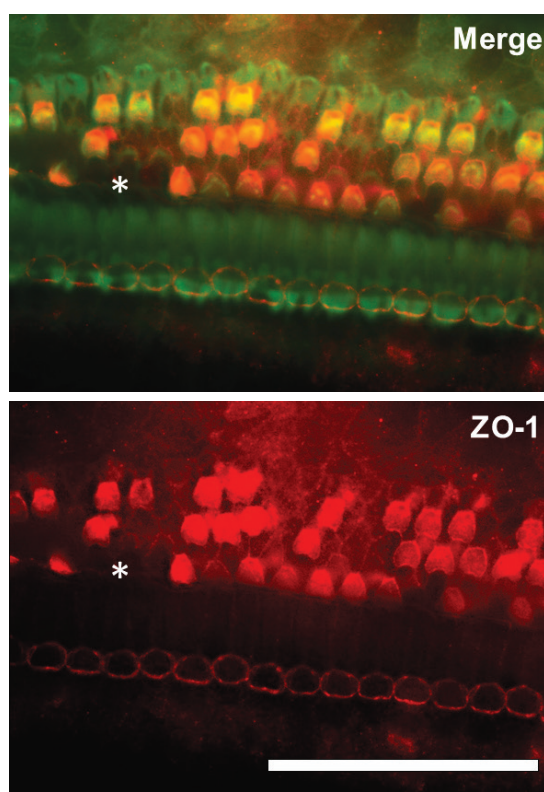

CD (-) "control"
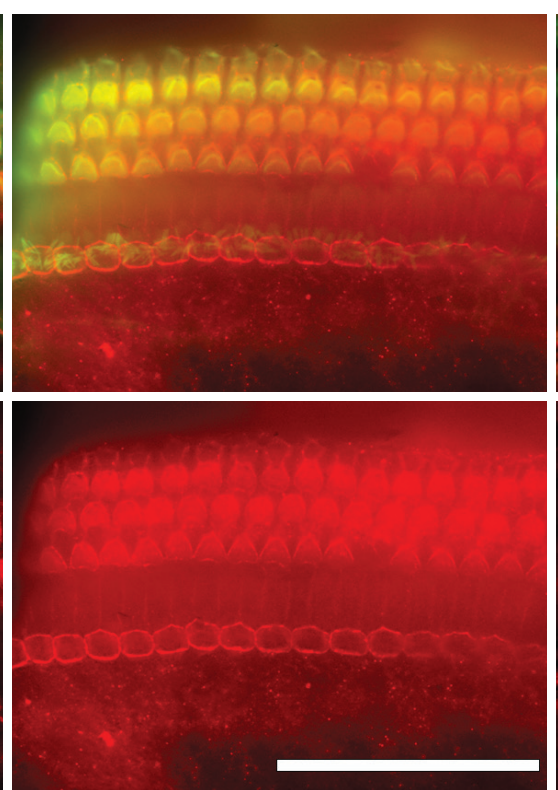

Ebselen $+C D$
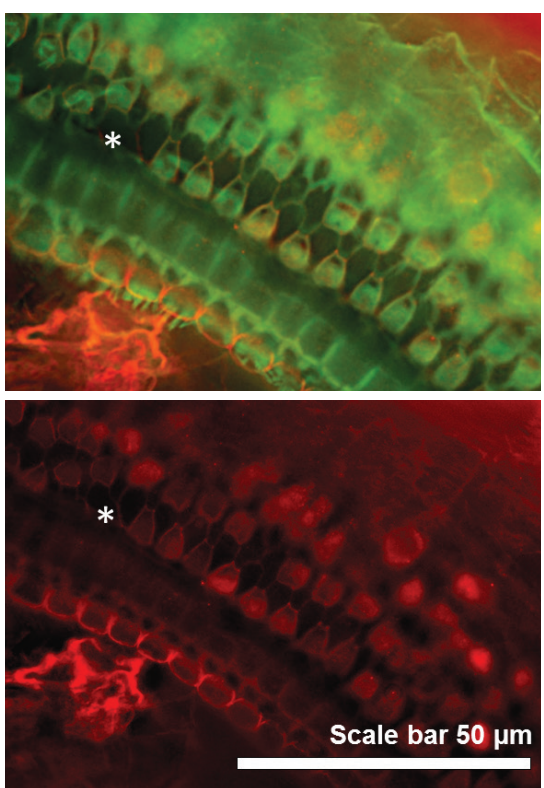

Fig. 3. Epi-fluorescence analysis of whole-mounts of the organ of Corti stained for ZO-1 and actin. Columns are organized as in figures 2. Tight junctional complexes have a similar appearance in OHCs in all 3 groups. Asterisks indicate the area where outer hair cells are replaced by phalangeal scars formed by supporting cells.

Fig. 4. Comparison of right-left symmetry of the transition zone location, where loss of $\mathrm{OHC}$ area transitioned into an area with preserved OHCs. HPBCD only group ears all exhibited symmetric hair cell transition areas from loss to survival, whereas combination group of ebselen and $\mathrm{HP} \beta C D$ demonstrates an asymmetric transition from $\mathrm{OHC}$ loss to survival in $20 \%$ of the animals.

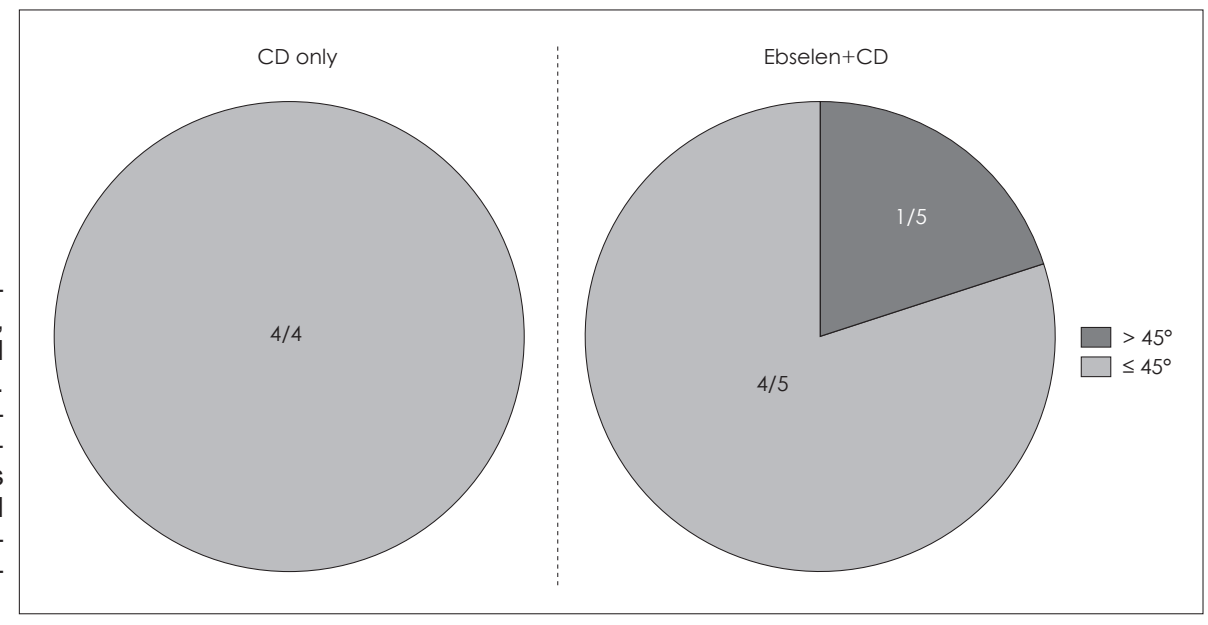

loss after systemic administration of $\mathrm{HP} \beta C D$ in mice, and showed that the addition of ebselen to HP $\beta C D$ treatment did not provide any functional and morphological rescue. Ebselen is one of many well-known anti-oxidants that show protective effects against various inducers of oxidative stress. Ebselen reduces ROS after ototoxic insults caused by cisplatin [12] and after acoustic overexposure [10]. It is not clear why ebselen did not ameliorate the cytotoxic effect of HP $\beta C D$ in this study. The observations add to previous data suggesting that the pathophysiologic mechanism of HP $\beta C D$ is not related to the formation of ROS [5] and suggest that additional work is needed to assess changes to mitochondrial bioenergetics [13] and other aspects of stress pathway effects that may yet be related to HP $\beta C D$. Still, according to the data of current study and former, measurement of ROS products using proper analytic tools such as DCF-DA or characterizing the downstream molecular product of ROS has not been performed. Therefore we highly recommend further analysis using such methods and using the other powerful ROS scavengers.

Another possible mechanism was the deterioration of tight junctions in the sensory epithelium. The loss of junctional integrity across the sensory epithelium can cause hair cell damage. Results from the present study revealed that there was no persistent loosening or disruption of tight junction complexes in the damaged auditory epithelium. The dramatic effects of methyl- $\beta$-cyclodextrin on tight junction integrity in model 
systems recovers rapidly after removal of the drug, with nearly full recovery in 24 hours [14]. However, we cannot rule out transient changes in junctions that occurred at earlier stages but recovered by the time of the analysis. Although cochlear damage induced by HP $\beta C D$ seems not to be related to chronic disorganization of tight junctional complexes as a main damage mechanism, but we cannot rule out acute damaging effects immediately after drug injection. This limitation could be recovered by adopting the functional permeability test such as Evans blue tracer assay $[15,16]$ which is not affected by timeline of experiments. As such, more work is needed to fully understand the pharmacokinetics of $\mathrm{HP} \beta C D$ in the cochlear duct and the resulting molecular events that ultimately lead to OHC death.

Similar to cochlear pathology induced by other ototoxic drugs and presbycusis, HP $\beta C D$ showed a base to apex gradient for drug susceptibility. This gradient may reflect pharmacokinetics and cochlear uptake mechanisms [17]. However, a recent study using direct drug delivery in the guinea pig also showed the basal susceptibility and suggested that the apex may be somewhat resistant [18].

The pattern of hair cell loss after systemic administration of $\mathrm{HP} \beta C D$ was quite different from characteristics of cellular damage induced by other ototoxic agents such as cisplatin or gentamicin. Systemic administration of most ototoxic drugs results in the change of hearing function at multiple frequencies, which corresponds well with the pathology of hair cell loss throughout the cochlea, even when the base is more sensitive [19]. It is unclear why cochlear damage by HP $\beta C D$ presents with such an abrupt cut-off point transitioning between damaged and undamaged hair cells. Several cochlear components show a gradient in expression or organization from base to apex, but none presents with such an abrupt transition. An abrupt transition between areas of hair cell presence versus compete loss has been shown in a mutant mouse [20] but the point of transition kept moving apically with time, and the mechanism is not easily correlated with the ototoxicity reported here.

This study has limitations as follows. First, administration protocol of HP $\beta C D$ based on experimental methods of previous studies $[3,5]$ was applied. With the different concentration and different delivery route result might be different and further studies using smaller or larger concentration or using different route of delivery should be conducted. Second, there were animals which were not affected by HP $\beta C D$. We believe that it may be due to slight variations in delivery method, not animal sensitivity. Additional study to demonstrate the characteristics of these unaffected animals may be required.

In conclusion, HP $\beta C D$-induced hearing loss show a histologic pattern of hair cell loss with abrupt transition from nor- mal to traumatized region, outer hair cell-restricted loss, and disorganization of prestin in remaining cells. The lesion was not minimized histologically or functionally by ebselen cotreatment. Tight junctional complexes were not affected by HP $\beta C D$ treatment. Further studies regarding pathophysiologic mechanism of HP $\beta C D$ may provide important clues for preventing ototoxicity in NPC patients treated with HP $\beta C D$.

\section{Acknowledgments}

This work was supported by the R. Jamison and Betty Williams Professorship to Y.R. and by the Dana's Angels Research Trust, Hide and Seek Foundation, and The Andrew Coppola Foundation as part of the SOAR-NPC (Support of Accelerated Research for NiemannPick type C Disease) to R.K.D. and by the National Institute on Deafness and Other Communication Disorders, National Institutes of Health (P30-DC05188).

\section{Conflicts of interest}

The authors have no financial conflicts of interest.

\section{REFERENCES}

1) Liu B, Turley SD, Burns DK, Miller AM, Repa JJ, Dietschy JM. Reversal of defective lysosomal transport in NPC disease ameliorates liver dysfunction and neurodegeneration in the npc1-/- mouse. Proc Natl Acad Sci U S A 2009;106:2377-82.

2) Pontikis CC, Davidson CD, Walkley SU, Platt FM, Begley DJ. Cyclodextrin alleviates neuronal storage of cholesterol in NiemannPick C disease without evidence of detectable blood-brain barrier permeability. J Inherit Metab Dis 2013;36:491-8.

3) Crumling MA, Liu L, Thomas PV, Benson J, Kanicki A, Kabara L, et al. Hearing loss and hair cell death in mice given the cholesterolchelating agent hydroxypropyl- $\beta$-cyclodextrin. PLoS One 2012;7: e53280.

4) Takahashi S, Homma K, Zhou Y, Nishimura S, Duan C, Chen J, et al. Susceptibility of outer hair cells to cholesterol chelator 2-hydroxypropyl-beta-cyclodextrine is prestin-dependent. Sci Rep 2016;6:21973.

5) Cronin S, Lin A, Thompson K, Hoenerhoff M, Duncan RK. Hearing loss and otopathology following systemic and intracerebroventricular delivery of 2-hydroxypropyl-beta-cyclodextrin. J Assoc Res Otolaryngol 2015;16:599-611.

6) Ward S, O'Donnell P, Fernandez S, Vite CH. 2-hydroxypropyl-beta-cyclodextrin raises hearing threshold in normal cats and in cats with Niemann-Pick type C disease. Pediatr Res 2010;68:52-6.

7) Huth ME, Ricci AJ, Cheng AG. Mechanisms of aminoglycoside ototoxicity and targets of hair cell protection. Int J Otolaryngol 2011; 2011:937861.

8) Zholudeva LV, Ward KG, Nichols MG, Smith HJ. Gentamicin differentially alters cellular metabolism of cochlear hair cells as revealed by $\mathrm{NAD}(\mathrm{P}) \mathrm{H}$ fluorescence lifetime imaging. J Biomed Opt 2015;20:051032.

9) Tabuchi K, Nishimura B, Nakamagoe M, Hayashi K, Nakayama M, Hara A. Ototoxicity: mechanisms of cochlear impairment and its prevention. Curr Med Chem 2011;18:4866-71.

10) Pourbakht A, Yamasoba T. Ebselen attenuates cochlear damage caused by acoustic trauma. Hear Res 2003;181:100-8.

11) Takumida M, Popa R, Anniko M. Free radicals in the guinea pig inner ear following gentamicin exposure. ORL J Otorhinolaryngol Relat Spec 1999;61:63-70.

12) Kim JB, Jung JY, Ahn JC, Rhee CK, Hwang HJ. Antioxidant and anti-apoptotic effect of melatonin on the vestibular hair cells of rat utricles. Clin Exp Otorhinolaryngol 2009;2:6-12.

13) Ziolkowski W, Szkatula M, Nurczyk A, Wakabayashi T, Kaczor JJ, 
Olek RA, et al. Methyl-beta-cyclodextrin induces mitochondrial cholesterol depletion and alters the mitochondrial structure and bioenergetics. FEBS Lett 2010;584:4606-10.

14) Francis SA, Kelly JM, McCormack J, Rogers RA, Lai J, Schneeberger EE, et al. Rapid reduction of MDCK cell cholesterol by methyl-beta-cyclodextrin alters steady state transepithelial electrical resistance. Eur J Cell Biol 1999;78:473-84.

15) Bors L, Tóth K, Tóth EZ, Bajza Á, Csorba A, Szigeti K, et al. Agedependent changes at the blood-brain barrier. A comparative structural and functional study in young adult and middle aged rats. Brain Res Bull 2018;139:269-77.

16) Li X, Shi X, Qiao Y, Xu K, Zeng L, Wang C, et al. Observation of permeability of blood-labyrinth barrier during cytomegalovirus-induced hearing loss. Int J Pediatr Otorhinolaryngol 2014;78:995-9.
17) Wu WJ, Sha SH, Schacht J. Recent advances in understanding aminoglycoside ototoxicity and its prevention. Audiol Neurootol 2002; 7:171-4.

18) Lichtenhan JT, Hirose K, Buchman CA, Duncan RK, Salt AN. Direct administration of 2-Hydroxypropyl-Beta-Cyclodextrin into guinea pig cochleae: effects on physiological and histological measurements. PLoS One 2017;12:e0175236.

19) Kuduban O, Kucur C, Sener E, Suleyman H, Akcay F. The role of thiamine pyrophosphate in prevention of cisplatin ototoxicity in an animal model. ScientificWorldJournal 2013;2013:182694.

20) Green KL, Swiderski DL, Prieskorn DM, DeRemer SJ, Beyer LA, Miller JM, et al. ACEMg diet supplement modifies progression of hereditary deafness. Sci Rep 2016;6:22690. 